\title{
THE DERIVATION OF ALGEBRAIC INVARIANTS BY TENSOR ALGEBRA*
}

\author{
BY C. M. CRAMLET $\dagger$
}

1. Review of Elements of Tensor Algebra. The few simple laws of tensor algebra offer a basis for a very natural approach to the theory of algebraic invariants. In the study of algebraic invariants a ground form is chosen as, for example, the bilinear $n$-ary form $a_{\rho \sigma} u^{\rho} v^{\sigma} \ddagger$ and the quantities $u^{r}$ and $v^{s}$ are transformed linearly by cogredient transformations such as

$$
\bar{u}^{r}=u^{\rho} q_{p}{ }^{r} .
$$

If the equivalent transformed form be now written $\bar{a}_{\rho \sigma} \bar{u}^{\rho} \bar{v}^{\sigma}$, the transformation equations for the $a$ 's are

$$
\bar{a}_{r s}=a_{\rho \sigma} p_{r} p_{s}^{\sigma},
$$

where the $p$ 's satisfy the equations

$$
p_{s}^{r} q_{\rho}^{r}=p_{\rho}^{r} q_{s}{ }^{p}=\delta_{s}^{r}, \quad(=0, r \neq s ;=1, r=s),
$$

or what is equivalent, $q_{s}{ }^{r}$ is equal to the cofactor of $p_{s}{ }^{r}$ in the determinant $\left|p_{s}^{r}\right|$, divided by this determinant. In tensor algebra the two equations of transformation (1) and (2) illustrate two types of tensors, that is, sets of ordered functions which are transformed in this linear manner. The set of quantities $u^{\rho}$ is called a contravariant tensor of rank 1 since the transformed quantities are each expressed linearly in the $q$ 's. The set of quantities $a_{r s}$ with two lower indices

* Presented to the Society, February 26, 1927, except $\$ 2$.

† National Research Fellow.

$\ddagger$ Repeated Greek letters are summed from 1 to $n$. Such a form is ordinarily written $a_{\rho \sigma} x^{\rho} y^{\sigma}$. In the theory of surfaces, with the constant values at a point, of a given tehsor such as $a_{r s}$ may be associated arbitrary vectors, in this case two, $u^{r}$ and $v^{s}$. Thus when a general transformation of coordinates is made the vectors are transformed linearly, so the theory of algebraic invariants is applicable at the point. 
illustrate a covariant tensor of rank 2. These terms covariant and contravariant are used differently in the classical theory of invariants. Here the terminology of tensor algebra will be used.

The three laws of tensor algebra which follow directly from the defining transformation equations are as follows.

(a) The sum of two tensors of the same kind is a tensor of that kind.

(b) The (outer) product of two tensors is a tensor whose rank is the sum of the ranks of these tensors. For example, $a_{r s} v^{t}$ is a mixed tensor covariant of rank 2 and contravariant of rank 1 . In the notation $T_{r s}{ }^{t}$, the transformation law is

$$
\bar{T}_{r s}^{t}=T_{\rho \sigma}^{\tau} q_{\tau}^{t} p_{r}^{\rho} p_{s}^{\sigma} \text {. }
$$

(c) If a covariant and contravariant index of a mixed tensor such as $T_{r s}{ }^{t}$ are set equal and summed, as the notation $T_{r \alpha}^{\alpha}$ indicates, a tensor is obtained which has its contravariant and covariant rank each decreased by one. The proof consists in setting $t=s=\alpha$ in the equation of (b) above, and making use of (3), from which we find

$$
\bar{T}_{r \alpha}^{\alpha}=T_{\rho \sigma}^{\tau} q_{\tau}^{\alpha} p_{r}^{\rho} p_{\alpha}^{\sigma}=T_{\rho \sigma}^{\tau} p_{r}^{\rho} \delta_{\tau}^{\sigma}=T_{\rho \alpha}^{\alpha} p_{r}{ }^{\rho} .
$$

This is the transformation equation defining a covariant vector. The expression $a_{\rho \sigma} u^{\rho^{\sigma}}$ having no unsummed indices is called a scalar. The process is called contraction or inner multiplication and the contracted or summed indices are said to cancel.

The symbol $\delta_{s}{ }^{r}$ which occurs in (3) may be made a tensor by assigning the law of transformation to it that its indices suggest. Then $\bar{\delta}_{s}^{r}=\delta_{\sigma}^{\rho} p_{s}^{\sigma} q_{\rho}^{r}=p_{s}^{\rho} q_{\rho}^{r}=\delta_{s}^{r}$, by (3) and the new components are exactly equal to the old components. In general therefore the tensor defined by the equation

$$
\gamma_{s_{1} \cdots s_{k}}^{r_{1} \cdots r_{k}}=B^{1} \delta_{s_{1}}^{r_{1}} \delta_{s_{2}}^{r_{2}} \cdots \delta_{s_{k}}^{r_{k}}+B^{2} \delta_{s_{1}}^{r_{2}} \delta_{s_{2}}^{r_{1}} \cdots \delta_{s_{k}}^{r_{k}}+\cdots
$$

in which every permutation of the set $r$ appears and the $B$ 's are arbitrary scalars, possesses this property. It has been 
proved* moreover that there are no other invariant tensors. The tensor $\delta_{s_{1} \ldots s_{k}}^{r_{1} \ldots r_{k}}$, obtained from (4) by assigning $B$ the values \pm 1 according as the permutation on $r$ of the $\delta$ 's of which $B$ is the coefficient is even or odd, since it transforms by invariance will (as does $\gamma_{s_{1} \ldots s_{k}}^{\gamma_{1} \ldots r_{k}}$ ) ) satisfy the equation

$$
\delta_{s_{1} \cdots s_{k}}^{r_{1} \cdots r_{k}}=\delta_{\sigma_{1} \cdots \sigma_{k}}^{\rho_{1} \cdots \rho_{k}} q_{s_{1}}^{\sigma_{1}} \cdots q_{s_{k} p_{\rho_{1}}}^{\sigma_{k} r_{1}} \cdots p_{\rho_{k}}^{r_{k}} \text {. }
$$

Setting $k=n$ and $r_{1} \cdots r_{n}=1 \cdots n$, this becomes

$$
\delta_{s_{1} \cdots s_{n}}^{1 \cdots n}=\delta_{\sigma_{1} \cdots \sigma_{k}}^{1 \cdots n} p_{s_{1}}^{\sigma_{2}} \cdots p_{s_{n}}^{\sigma_{n}} \cdot\left|q_{s}^{r}\right| \text {. }
$$

This is usually written as

$$
\epsilon_{s_{1}} \cdots s_{n}=\epsilon_{\sigma_{1}} \cdots \sigma_{n} p_{s_{1}}^{\sigma_{1}} \cdots p_{s_{n}}^{\sigma_{n}} \cdot\left|q_{s}{ }^{r}\right|,
$$

and is called a covariant tensor $\dagger$ density of weight -1 . This equation represents its transformation equation and shows these important facts: (1) it is invariant under a transformation of coordinates; (2) the indices are of tensor character, that is, indicate a certain law of transformation; (3) a factor $\Delta^{-1}$ must be introduced after a transformation of coordinates. Similarly from (5), setting the indices $s$ equal to $1 \cdots n$, we have

$$
\epsilon^{r_{1} \cdots r_{n}}=\epsilon^{\rho_{1} \cdots \rho_{n}} q_{\rho_{1}}^{r_{1}} \cdots q_{\rho_{n}}^{r_{n}} \cdot \Delta,
$$

and $\epsilon^{r_{1} \ldots r_{n}}$ is called a contravariant tensor density of weight 1 .

2. Proof of the Fundamental Theorems. $\ddagger$ It will now be

* P. Franklin, Philosophical Magazine, vol. 45, p. 998; C. M. Cramlet, this Bulletin, vol. 32 (1926), p. 212; T. Y. Thomas, Annals of Mathematics, vol. 27, No. 4 (1926), p. 548; C. M. Cramlet, Tôhoku Mathematical Journal, vol. 28, Nos. 3, 4 (1927), p. 242.

$\dagger$ See Veblen, Invariants of Quadratic Differential Forms, Cambridge Tract No. 24, 1927, Chapter I and Chapter II, \$14.

$\ddagger$ The problem of $\$ 2$ was discussed in a series of conferences with Professor O. Veblen and Mr. W. Flexner. Thanks are due to Professor Veblen for constructive criticism which was helpful for a clear formulation of the problem. Mr. Flexner has worked out a proof of this problem by the use of the Cayley operator. The novel feature of his proof is the fact that the sy mbolic method is not used. 
shown that the determination of all algebraic invariants reduces to an exercise of the three laws of tensor algebra.

Let us suppose that we have a system of forms determined by the coefficients (or covariant tensors) $a_{r_{1} \ldots r_{h}}, b_{r_{h+1} \ldots r_{i}}$, $\cdots, c \ldots r_{k}$. Assuming the theorems, which depend upon elementary considerations on the arbitrariness of these forms, that only functions homogeneous in each of the tensors separately need be considered, we know that a typical term of an algebraic invariant is

$$
A_{r_{1} \cdots r_{k}} \equiv a_{r_{1}} \cdots r_{h} b \cdots r_{i} \cdots c \cdots r_{k} \text {, }
$$

and the invariant is representable as

$$
I=K^{\rho_{1}} \cdots \rho_{k} A_{\rho_{1}} \cdots \rho_{k}
$$

the $K$ 's being undetermined constants. An algebraic invariant must by definition be transformed according to the equation $\bar{I}=I \cdot F$, where $\bar{I}$ is exactly the same function of the coefficients of the transformed forms as is $I$ of the original forms. Assuming further the well known theorem, which is a consequence of the assumption that $I$ is rational, that $F$ is an integral power of the modulus of the transformation, say $\Delta^{w}$ where $\Delta=\left|p_{j}^{i}\right|$, we may write this equation in the form

$$
K^{\rho_{1} \cdots \rho_{k}} \bar{A}_{\rho_{1} \cdots \rho_{k}}=K^{\rho_{1} \cdots \rho_{k}} A_{\rho_{1} \cdots \rho_{k}} \Delta^{w}
$$

On the other hand, since $A$ is a tensor, we have

$$
K^{\rho_{1}} \cdots \rho_{k} \bar{A}_{\rho_{1}} \cdots \rho_{k}=K^{\rho_{1} \cdots \rho_{k}} A_{\sigma_{1}} \cdots \sigma_{h} p_{\rho_{1}}^{\sigma_{1}} \cdots p_{\rho_{k}}^{\sigma_{k}} .
$$

The right members of these equations are identically equal for arbitrary $p$ 's; hence $k=w n$. Multiplying (6) by $\Delta$ and (9) by $w \epsilon$ 's, we find, by direct substitution of this new (6) into this new (9),

$$
\begin{aligned}
\epsilon_{r_{1}} \cdots r_{n} \cdots \epsilon & \cdots r_{k} K^{\rho_{1} \cdots \rho_{k}} \bar{A}_{\rho_{1}} \cdots \rho_{k} \\
& =K^{\sigma_{1} \cdots \sigma_{k}} A_{\sigma_{2} \cdots \sigma_{k}} \epsilon_{\rho_{1}} \cdots \rho_{n} \cdots \epsilon \cdots \rho_{k} p_{r_{1}}^{\rho_{1}} \cdots p_{r_{k}}^{\rho_{k}}
\end{aligned}
$$


Substituting in this

$$
A_{\sigma_{1}} \cdots \sigma_{k}=\bar{A}_{\tau_{1}} \cdots \tau_{k} q_{\sigma_{1}}^{\tau_{1}} \cdots q_{\sigma_{k}}^{\tau_{k}}
$$

we have

$$
\begin{aligned}
& \tau_{1} \bar{A} \cdots \tau_{k}\left[\epsilon_{r_{1}} \cdots r_{n} \cdots \epsilon \cdots r_{k} K^{\tau_{1} \cdots \tau_{k}}\right. \\
& \left.\quad-\epsilon_{\rho_{1}} \cdots \rho_{k} \cdots \epsilon \cdots \rho_{k} K^{\sigma_{1}} \cdots \sigma_{k} q_{\sigma_{1}}^{\tau_{1}} \cdots q_{\sigma_{k}}^{\tau_{k}} p_{r_{1}}^{\rho_{1}} \cdots p_{r_{k}}^{\rho_{k}}\right]=0 .
\end{aligned}
$$

If $\bar{A}$ is an arbitrary tensor its coefficient must vanish. But $A$ may be a product of tensors, in which case the terms of the product are interchangeable, or, one of the tensors may determine a $p$-ic in which case its indices are interchangeable. There is no loss of generality in the invariant $I$ defined by equation (8) if the $K$ 's are assumed to have the same symmetry as the $A$ 's. This amounts to replacing the constants associated with equal terms by their average. We may now set coefficients of $\bar{A}$ equal to zero as in the case where $\bar{A}$ is wholly arbitrary. The vanishing of these coefficients expresses the conditions that $\epsilon_{r_{1}} \ldots r_{n} \cdots \epsilon \ldots r_{k} K^{\tau_{1}} \ldots \tau_{k}$ be an invariant tensor of the rank indicated by the indices, that is a tensor having identical components in all systems of coordinates. Using the theorem of $\$ 1$ embodied in (4), we find $\epsilon_{r_{1}} \cdots r_{n} \cdots \epsilon \cdots r_{k} K^{\rho_{1}} \cdots \rho_{k}=B^{1} \delta_{r_{1}}^{\rho_{1}} \delta_{r_{1}}^{\rho_{2}} \cdots \delta_{r_{k}}^{\rho_{k}}+B^{2} \delta_{r_{1}}^{\rho_{2}} \delta_{r_{2}}^{\rho_{1}} \cdots \delta_{r_{k}}^{\rho_{k}}+\cdots \cdot$ Multiplying through by $\epsilon^{r_{1} \ldots r_{n}} \ldots \epsilon^{\cdots r_{k}}, r$ summed, we have (13) $(n !)^{w} K^{\rho_{1} \cdots \rho_{k}}=B^{1} \epsilon^{\rho_{1} \rho_{2} \cdots \rho_{n}} \cdots \epsilon^{\cdots \rho_{k}}+B^{2} \epsilon^{\rho_{1} \rho_{2} \cdots \rho_{n}} \cdots \epsilon^{\cdots \rho_{k}}+\cdots$

Since $k=w n$, each term contains $w \epsilon$ 's and the $\rho$ 's appear in all possible permutations. We have thus found that for $I$ of (8) to be an invariant it is necessary that $K^{\rho_{1}} \cdots \rho_{k}$ be of the form (13). It is obviously sufficient, for when each term of (13) with the $B$ 's arbitrary is multiplied by inner multiplication with $A_{\rho_{1} \ldots \rho_{k}}$ the tensor indices cancel by inner multiplication according to tensor law (c), $\$ 1$, and, since $\epsilon^{\rho_{1} \ldots \rho_{n}}$ is a tensor density of weight one, each term gives rise to a scalar density of weight $w$. Those solutions that do not satisfy the symmetry conditions imposed on $K$, 
that is, those which have two antisymmetric indices on an $\epsilon$ summed with two symmetric indices on an $A$, will vanish identically. We thus have the following theorem.

THEOREM 1. An algebraic invariant of weight $w$ is formed from a given system of covariant tensors by forming by outer multiplication a tensor $A$ of rank wn and multiplying this by inner multiplication by $w \epsilon$ 's, associating the indices of the $\epsilon$ 's in any manner whatever with the indices of $A$. Moreover these constitute all algebraic invariants that can be formed from the given covariant tensors.

The extension to the following general theorem is immediate.

THEOREM 2. From a given system of covariant and contravariant tensors, a tensor $A_{r_{1} \cdots r_{k}}^{s_{1} \cdots s_{l}}$ may be formed by outer and inner multiplication. In this tensor, $k$ and $l$ are multiples of $n$, say, $k=w_{1} n, l=w_{2} n$ and $w=(k-l) / n$, all letters representing integers. All algebraic invariants may be formed by multiplying by inner multiplication $w_{1}$ of the contravariant $\epsilon^{\prime} s, \epsilon^{r_{1} \ldots r_{n}}$ and $w_{2}$ covariant $\epsilon^{\prime} s, \epsilon_{r_{1}} \ldots r_{n}$. A few illustrations of the methods here described will be given.

3. Algebraic Invariants of a System of Linear Forms. It will require $n$ tensors such as $a_{r}$ to build up an $n$th rank tensor by outer multiplication. These $n$ tensors can be represented by $a_{r \mid s}$. The bar is used to indicate that the preceding $r$ does not designate a tensor character. The only possible algebraic invariant is $\epsilon^{\rho_{1}} \cdots{ }^{\rho n} a_{r_{1} \mid \rho_{1}} \cdots a_{r_{n} \mid \rho n} . *$ This is merely the determinant of the system of forms. For a set of vectors $\left.u_{r \mid}\right|^{8}$ the algebraic invariant of weight -1 is

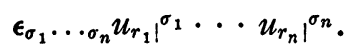

* The non-tensor range is alternating here. The number of alternating ranges associated with $n$ elements is always even. See my paper, Applications of the determinant and permanent tensors to determinants of general class and allied tensor functions, American Journal, vol. 49, No. 1 (January, 1927), p. 87. 
4. Symmetry Tensors. When two or more indices of an element are summed with indices of the same $\delta$ or $\epsilon$ there results an algebraic invariant whose vanishing expresses a symmetry condition on the ground form. For example, if the vectors in the bilinear form $a_{\rho \sigma} u^{\rho} v^{\sigma}$ have but two components ( $n=2$, the binary bilinear form), we may form the algebraic invariant $\delta_{r s}^{\rho \sigma} a_{\rho \sigma}$. The vanishing of this indicates that $a_{r s}=a_{s r}$ in all coordinate systems. The property remains true for any $n$ but then we have a tensor that is not an algebraic invariant. The property of being skew symmetric is a property that carries over to any new form yet the condition is not expressible as an algebraic invariant but only as a tensor. This condition is expressed by the tensor equation

$$
\pi_{r s}^{\rho \sigma} a_{\rho \sigma}=0,\left(\pi_{r s}^{\rho \sigma}=\delta_{r}^{\rho} \delta_{s}^{\sigma}+\delta_{r}^{\sigma} \delta_{s}^{\rho}\right), \quad \text { or } \quad a_{r s}=-a_{s r} .
$$

In a new coordinate system this becomes $\pi_{r s}^{\rho \sigma} \bar{a}_{\rho \sigma}=0$, from which we find $\bar{a}_{r s}=-\bar{a}_{s r}$. The method of obtaining other types of symmetry conditions for tensors with the use of the $\delta$ or $\gamma$ tensors is apparent.*

5. Bilinear Forms. With the two-index tensor $a_{r s}$ the following invariant of weight one may be formed if $n$ is even (equal to $2 i$, say):

$$
\epsilon^{\rho_{1} \sigma_{1}} \cdots \rho_{i} \sigma_{i} a_{\rho_{1} \sigma_{1}} \cdots a_{\rho_{i} \sigma_{i}} .
$$

If we seek the invariants of weight two, the degree must equal $n$, for example

$$
\epsilon^{\rho_{1}} \cdots \rho_{n} \epsilon^{\sigma_{1}} \cdots \sigma_{n} a_{\rho_{1} \sigma_{1}} \cdots a_{\rho_{n} \sigma_{n}} .
$$

The set of indices either $\rho$ or $\sigma$ on the elements is called a range. If the tensor is not symmetric the indices of an $\epsilon$ need not be summed with a range. There will then be many invariants like (15). If, however, they are summed with a range, the second $\epsilon$ merely multiplies the invariant by $n !$.

6. Systems of Bilinear Forms. Explicit expressions for a

*Weyl, Space, Time, Matter, 4th edition, pp. 57, 58. 
system of bilinear forms are readily written. Represent the system by

$$
\phi_{q \mid}=a_{q \mid \rho \sigma} u^{\rho v^{\sigma}}, \quad(q=1,2, \cdots) .
$$

The following is a set of invariants of the forms:

$$
\epsilon^{\rho_{1}} \cdots \rho_{n} \epsilon^{\sigma_{1}} \cdots \sigma_{n} a_{q_{1} \mid \rho_{1} \sigma_{1}} \cdots a_{q_{n} \mid \rho_{n} o_{n}} .
$$

The $q$ 's may be distinct or repeated (see reference in \$3) and any of the elements may be outer products of elements of linear forms.

7. Trilinear Forms. With a given tensor $a_{r s t}$ it is only necessary to construct inner tensor products with the tensors $\epsilon^{\rho_{1} \ldots \rho_{n}}$ so that all indices on the elements are summed. All such are algebraic invariants and all algebraic invariants are thus acquired. To illustrate in detail a simple case, choose $n=2$ and require umbral letters on an $\epsilon$ to be associated with indices of a range. Such invariants will also be invariants of the cubic, for which $a_{r s t}$ is symmetrical. Thus consider the invariant of degree 4 and weight 6 of the binary cubic

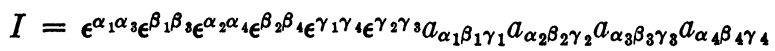

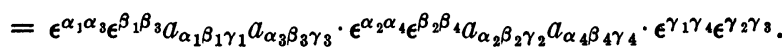

The product of the first four quantities in this equation is a cubic determinant so the indices $\gamma_{1}$ and $\gamma_{3}$ are a permanent range. We may represent this product by $A_{\gamma_{1} \gamma_{\xi}}$. The whole theory of algebraic invariants might be translated into theorems regarding inner products of higher determinants. Further details of evaluating this invariant will be carried out:

$$
I=\epsilon^{\gamma_{1} \gamma_{4}} A_{\gamma_{1} \gamma_{3}} A_{\gamma_{4} \gamma_{2}} \epsilon^{\gamma_{2} \gamma_{3}} .
$$

The first three terms of this are again a determinant, hence the range $\gamma_{2} \gamma_{3}$ is alternating and yields when multiplied innerly with $\epsilon^{\gamma_{2} \gamma_{3}}, I=2 ! \epsilon^{\gamma_{1} \gamma_{4}} A_{\gamma_{1} \gamma_{3}} A_{\gamma_{4} \gamma_{2}}$. This invariant is determined by the particular manner in which the indices 
of an $\epsilon$ are associated with the indices of the elements; thus schematically we may write 17 as

$$
I=(13)(13)(24)(24)(14)(23)=(13)^{2}(24)^{2}(14)(23) \text {. }
$$

This expression is 2 ! times the invariant represented in the same manner by the symbolic method.

8. Conclusion. We have here laid down a basis for a development of the theory of algebraic invariants which is applicable to tensors of any type. Transition can be made to the symbolic method which now becomes established without the use of ideal vectors. The computation seems particularly more simple than for the symbolic method.

The $\epsilon$ symbols have been used throughout and the number of indices on an $\epsilon$ was always the same as the number of components of a vector in $n$-space or as the number of variables used to represent a point in $(n-1)$-space in homogeneous coordinates. If in any of the formulas we replace an $\epsilon^{r_{1} \ldots r_{n}}$ or $\epsilon_{s_{1} \ldots s_{n}}$ by a $\delta_{s_{1} \ldots s_{k}}^{r_{1} \ldots r_{k}}$ and allow $k$ to be less than $n$, each algebraic invariant becomes an alternating tensor which has 1 distinct component for $k=n$ and more components for $k<n$. This observation shows rather clearly the position algebraic invariants occupy in tensor algebra and again among alternating tensors. The reason that algebraic invariants have been of greater interest lies in the fact that the vanishing of an algebraic invariant gives but one condition while the vanishing of any other tensor is a much stronger condition since it gives rise to as many conditions as the tensor has distinct components.*

Princeton University

*F. L. Hitchcock has written a number of papers bearing upon the subject of this paper, in particular see $A$ new method in the theory of quantics, Journal of Mathematics and Physics, vol. 4 (1925), p. 238. If his view point is adopted in this paper, the results remain true if the indices associated with each $\epsilon$ and with the indices of $A$ that are multiplied innerly with this $\epsilon$ are cogredient, that is, we know that all invariants are obtained by these methods. Further we know that all such upon specialization by making all indices cogredient become algebraic invariants. Then, however, the question would still remain open as to whether these included all algebraic invariants. 